\title{
Processo de trabalho de gerência: uma revisão da literatura
}

\author{
MANAGEMENT WORK PROCESS: A FOCUS OVER ANALITICS' DIMENSIONS \\ PROCESSO DE TRABAJO DE GERENCIA: UN ENFOQUE SOBRE LAS DIMENSIONES DEL ANALISE
}

\author{
Regina Célia Ermel ${ }^{1}$, Lislaine Aparecida Fracolli ${ }^{2}$
}

\begin{abstract}
RESUMO
O objetivo deste artigo é identificar como o trabalho de gerência tem sido abordado nas publicações científicas. Tomou-se como objeto a produção bibliográfica sobre gerência produzida entre 1989 a 1999. A coleta de dados envolveu a busca nas bases de dados Medline e Lilacs. Os trabalhos encontrados foram analisados segundo a técnica de Análise de Conteúdo, proposta por Bardin. Os resultados mostraram que 38\% das publicações abordavam a dimensão organizacional da gerência; 34\% abordavam a dimensão técnica; $9 \%$ a dimensão politica e 19\% articulavam duas ou mais dessas dimensões. A análise da literatura nos indica a necessidade de desenvolver produções científicas que discutam a gerência como um trabalho político, técnico e organizacional.
\end{abstract}

\section{PALAVRAS-CHAVE}

Administração em enfermagem. Serviços de saúde. Gerência.

\begin{abstract}
The objective of this paper is to identify how the management work has been studied in scientific publications. The bibliography of management produced in 1989 to 1999 was analyzed. The date was collected in Medline and Lilacs data bases. The scientific publications founded, were analysed second the technique of Contents' Analysis, proposal by Bardin. The results showed that $38 \%$ described the organizational dimension of management; $34 \%$ described the technical dimension; $9 \%$ described the political dimension and 19\% articulated two or more of these dimensions. The analyses of the bibliography showed the necessity to increase scientific productions that discuss management as a technical,political and organizational work.
\end{abstract}

\section{KEYWORDS}

Nursing administration.

Health services.

Management.

\section{RESUMEN}

El objetivo de este articulo é identificar cómo el trabajo de gerencia ha sido estudiado en las publicaciones científicas. El objecto de análisis fue la producción bibliografica acerca de la gerencia entre los años de 1989 a 1999. La búsqueda fue hecha en las bases de datos Medline e Lilacs. Ls trabajos encuentrados fueron analisados segun la técnica de Analisis de Contenido, propuesta por Bardin. Los resultados mostraram que 38\% das publicaciones abordaban la dimensión organizacional de la gerencia; 34\% abordaban la dimensión técnica; 9\% abordaban la dimensión política e 19\% articulaban dos o más de estas dimensiones. El análisis de la literatura indicó la necesidad de desarrollarse producciones cientificas que discutan la gerencia como un trabajo político-técnico y organizacional.

\section{PALABRAS-CLAVE}

Administración en enfermería. Servicios de salud.

Gerencia.

\author{
1 Enfermeira. Mestranda \\ em Enfermagem em \\ Saúde Coletiva na \\ EEUSP. \\ rcermel@terra.com.br \\ 2 Enfermeira. Professora \\ Doutora do Depto. de \\ Enfermagem em Saúde \\ Coletiva da EEUSP. \\ lislaine@usp.br
}




\section{INTRODUÇÃO}

O processo de descentralização administrativa desencadeado pelo Sistema Único de Saúde (SUS), coloca a questão da tecnologia gerencial como uma preocupação fundamental para a implementação de um Sistema de Saúde hierarquizado, regionalizado e com efetiva participação social.

O processo de municipalização da saúde, faz parte do movimento de reforma administrativa do Estado brasileiro, e para o município significa concretamente a possibilidade de, a partir da saúde, reestruturar a gestão municipal em seu conjunto em uma perspectiva democrática, participativa, tecnicamente competente e gerencialmente eficiente.

A municipalização da saúde ocasiona um fortalecimento da autonomia político-gerencial dos municípios e uma elevação da sua capacidade técnico-operacional de planejamento, programação, controle gerencial e operacionalização de ações, voltadas ao enfrentamento dos problemas de saúde em territórios específicos. Este fato evidencia a importância das tecnologias de gerenciamento em saúde para os municípios pois, historicamente, estes funcionaram na área da saúde como executores de ações planejadas no âmbito federal e, portanto, não acumularam experiência em planejar, desenvolver e avaliar políticas de saúde, ou seja, em gerenciar autonomamente a saúde no seu espaço geopolítico.

O processo de trabalho de gerência da produção de serviços de saúde é considerado um instrumento potente para a efetivação de políticas públicas pois, o trabalho de gerência, é ao mesmo tempo, "condicionante" e "condicionado" pela forma como se organiza a produção dos serviços de saúde e consequentemente o modelo tecno-assistencial em curso $^{(1)}$.

Merhy ${ }^{(2)}$, em seus trabalhos, defende a idéia de que gerenciar serviços de saúde é atuar numa "dobra" entre a ação e o autogoverno dos sujeitos do trabalho em saúde. Esse autor diz que, gerenciar é menos capturar sujeitos para um trabalho e mais "construir" sujeitos. Sujeitos estes que possam ser criativos, éticos, tecnicamente competentes e associados a um projeto de saúde "em defesa da vida" das pessoas. Baseado nessas concepções, o autor sugere alguns "dispositivos" que, ao atuarem em determinados campos de gestão, podem operar mudanças nos serviços de saúde, considerando a existência de três dimensões no trabalho de gerência: o campo da política, o campo da organização e o campo dos processos de trabalho. Esse autor aposta que o cruzamento dessas dimensões (política, organizacional e processo de traba1ho), colocadas sob a ação de certos dispositivos de intervenção, capazes de atuar nos focos do gerir e do agir podem, como resultante final, alterar a lógica do cuidado à saúde e, consequentemente, o modelo de atenção, a partir do interior dos serviços de saúde.

Analisar o trabalho de gerência dos serviços de saúde, entendendo que este tem potencial para transformar o modelo de saúde a partir do seu momento mais operacional, isto é, a partir da produção do serviço, é um argumento suficiente para justificar a necessidade de pesquisas e de construção de saberes teórico-práticos que embasem a realização de processos gerenciais que reorganizem, os serviços e os sistemas de saúde, a partir de sua base.

A gerência de serviços de saúde tem sido, historicamente, uma prática realizada por enfermeiras, as quais têm utilizado para isso a sua capacidade de saber organizar e sistematizar rotinas, legitimando a hierarquia e a disciplina presentes nas instituições públicas. Contudo, falta à enfermeira conscientizar-se do potencial transformador de seu trabalho de gerência, considerando que através desse processo de trabalho ela pode introduzir instrumentos inovadores para reorganizar o cuidado e a divisão social do trabalho ${ }^{(3)}$.

Diante da importância que a questão do trabalho de gerência dos serviços de saúde tem assumido, atualmente, para o SUS e, historicamente, para a enfermagem e frente às mudanças conceituais ocorridas nesse campo científico, surge a importância de se identificar os padrões de gerência adotados na prática cotidiana dos serviços de saúde e as concepções teóricas que embasam estes padrões gerenciais, buscando visualizar se, estas práticas gerenciais, são construções inovadoras dos sujeitos da ação ou são normas instituídas pelos serviços e pelas políticas de saúde.

Desta maneira, o objetivo desta investigação é levantar produções bibliográficas sobre gerenciamento de serviços de saúde, produzida entre 1989 e 1999, ou seja, num período de dez anos, buscando identificar a natureza do conhecimento produzido sobre gerenciamento de serviços de saúde. 


\section{MÉTODO}

Este estudo se caracteriza como uma pesquisa bibliográfica. Os dados foram coletados através do levantamento das produções científicas, sobre o processo de trabalho de gerência, produzidas entre os anos de 1989 a 1999. As bases utilizados para a coleta de dados foram os bancos de dados MEDLINE e LILACS, a busca bibliográfica foi realizada utilizando-se como descritores as palavras ADMINISTRAÇÃO DE SERVIÇOS DE ENFERMAGEM, ADMINSTRAÇÃODE SISTEMAS DE SAÚDE, SAÚDE PUBLICA.

Para a organização das informações, contidas nas publicações científicas encontradas, foi utilizada a leitura flutuante dos resumos dos trabalhos, identificando-se o objeto, os objetivos do estudo e os resultados do mesmo, os dados foram registrados sob a forma de fichas de leitura. Para análise dos dados este estudo utilizou a técnica de Análise de Conteúdo, proposta por $\operatorname{Bardin}^{(4)}$.

A Análise de Conteúdo, pode ser definida como

\begin{abstract}
Um conjunto de técnicas de análise de comunicação visando obter, por procedimentos sistemáticos e objetivos de descrição do conteúdo das mensagens, indicadores (quantitativos ou não) que permitam a inferência de conhecimentos relativos às condições de produção/recepção destas mensagens ${ }^{(4)}$.
\end{abstract}

A Análise de Conteúdo na sua história mais recente, isto é, enquanto técnica de tratamento de dados considerada cientificamente, é caudatária das metodologias quantitativas, buscando sua lógica na interpretação cifrada do material de caráter qualitativo. Enfim, é uma técnica de pesquisa para descrição objetiva, sistemática e quantitativa do conteúdo manifesto das comunicações e tendo por fim interpretá-las. Os conteúdos temáticos, encontrados nos resumos dos trabalhos, eram categorizados segundo as dimensões política, organizacional e de processos de trabalho de gerência ${ }^{(2)}$.

A dimensão política, está sempre presente na gerência de serviços de saúde pois, gerenciar um serviço de saúde é, de alguma forma, implementar uma "dada" política pública de saúde assim, os trabalhos encontrados no levantamento bibliográfico que, descreviam ou questionavam a imple- mentação de políticas de saúde ou modelos tecnoassistencias através do gerenciamento de serviços, foram classificados nessa categoria.

A dimensão organizacional do trabalho de gerência, é aquela que revela as "normas", os "contratos" (explícitos ou não) que regem as relações entre os agentes do trabalho e entre estes e a instituição, assim estudos encontrados com a pesquisa bibliográfica e que apresentavam considerações sobre formas de gerenciar e características "normativas" das relações de trabalho nos serviços de saúde, foram alocados nessa categoria.

Com relação à dimensão do processo de trabalho de gerência, esta é definida como o lugar que representa a produção dos "atos" e "ações" de saúde, então, foram alocadas nesta dimensão as referências bibliográficas de estudos que discutiam as técnicas de gerência e as bases teóricas das mesmas.

\section{RESULTADOS E DISCUSSÃO}

No período de 1989 a 1999 foram encontrados 50 trabalhos que tomavam a questão da gerência como tema central. Dos trabalhos encontrados, $81 \%$ eram estudos que abordavam a temática gerência a partir de uma única dimensão e apenas $19 \%$ abordavam o trabalho de gerência através da articulação de duas ou mais das dimensões trazidas nesteestudo.

Dos trabalhos que abordavam o trabalho de gerência, apenas, por uma dimensão encontrou-se que $38 \%$ dos estudos abordavam a dimensão organizacional do gerenciamento; $34 \%$ abordavam a dimensão de gerência relacionada aos processos de trabalho e $9 \%$ dos trabalhos levantados abordavam a dimensão política do trabalho gerencial.

De certa forma era esperado que os estudos sobre gerenciamento estivessem centrados na dimensão organizacional (abordando principalmente a produção de técnicas de gerenciamento) pois, historicamente, o conhecimento sobre gerenciamento evoluiu seguindo o caminho de construção de tecnologias de gestão e de tecnologias para o entendimento das organizações enquanto espaços de trabalho.

As funções de gerência surgem pelo próprio exercício do trabalho cooperativo e no início do modo de produção capitalista a função da gerência se estabeleceu como uma
Processo de trabalho de gerência: uma revisão de literatura 
Regina Célia Ermel Lislaine Aparecida Fracolli forma de controle da produção ${ }^{(5)}$. De fato, o controle é o conceito fundamental da maioria das teorias e sistemas gerenciais. Na construção da sua teoria administrativa, Taylor esforçou-se para sistematizar conhecimentos relativos à organização do trabalho, tal sistematização de conhecimentos visava, sobretudo, aumentar a rentabilidade do trabalho fabril, numa fase de grande expansão do capitalismo $^{(6)}$.

Um outro grande pilar dos primórdios teóricos da administração é a proposta de Henri $\mathrm{Fayol}^{(6)}$. Desenvolvida numa perspectiva mais ampla, tal proposta enfocava a organização administrativa e a sistematização do trabalho do dirigente, sua concepção das funções do administrador, traduzida em cinco verbos - planejar, organizar, dirigir, coordenar e controlar - fundamentou, até recentemente, toda uma produção de conhecimento no campo da gerência.

$\mathrm{Na}$ construção histórica do seu corpo de conhecimento a Teoria Geral da Administração ${ }^{(6)}$, em meados do século XX, sofre uma significativa ampliação do seu objeto, passando a mesma a incorporar, a "organização" e não apenas o trabalho desenvolvido dentro dela. Nesse período os "saberes" constituídos junto à Teoria Geral da Administração se apresentaram sob duas formas: de um lado a ênfase no comportamento humano "dentro da organização" sob a influência da psicologia, enfocando aspectos de liderança, motivação e tomada de decisão, entre outros; e do outro lado, a "ênfase no ambiente", baseada nas idéias de Max Weber e Talcott Parsons, que se referiam respectivamente aos estudos da Burocracia e do Sistema Social ${ }^{7}$. A Teoria Geral da Administração, enquanto formulação teórica sobre gerenciamento, vem sendo objeto de análise em diversos estudos da área de saúde, estes estudos têm procurado desvendar o caráter ideológico da Teoria Geral da Administração em contraposição à singularidade da administração pública e a contribuição de outras escolas (como a marxiana) para o campo da gestão em saúde $^{(8)}$.

A análise dos resumos das publicações relativas à gerência dos serviços de saúde identificou, também, que os trabalhos que abordavam a gerência em uma dimensão de processo de trabalho exploravam essa dimensão, discutindo ferramentas que podiam ser utilizadas para tomada de decisões na área gerencial, com a finalidade de melhorar a qualidade dos servi- ços prestados; identificavam ações e elementos facilitadores e dificultadores da qualidade dos serviços oferecidos; forneciam subsídios para a criação e operacionalização dos sistemas de informação em saúde e em enfermagem; abordavam o uso da epidemiologia e da vigilância epidemiológica nos serviços locais de saúde como instrumento de gerenciamento; apresentavam a formulação de normas, procedimentos e rotinas como parte integrante do processo administrativo, traziam orientações quanto a forma de se organizar o trabalho para a implantação de unidades de saúde, apresentavam os saberes que deveriam subsidiar a execução do trabalho de gerência e experiências relativas a programas de ensino de gerenciamento desenvolvidos no âmbito da graduação em enfermagem ${ }^{(9-19)}$.

Tem sido amplamente discutido que as teorias gerenciais em uso, foram formuladas e desenvolvidas para serem aplicadas nos setores industriais e não em Serviços de Saúde, dessa forma, as dificuldades administrativas do setor de serviços se deve à ausência de formulações teóricas sobre gerência próprias para esse setor. Surge desse fato a necessidade de se construir e consolidar conhecimentos sobre gerência específicos para área de saúde ${ }^{(9)}$. Este estudo nos permitiu constatar que os profissionais de saúde têm se dedicado à construção desse conhecimento, sobre gerenciamento a ser aplicado, especificamente para a área da saúde .

O levantamento bibliográfico mostrou, também, que os trabalhos que abordavam, preferencialmente, a dimensão política do trabalho de gerência, de um modo geral, problematizavam a questão das formas de intervenção do Estado na sociedade, através de políticas sociais voltadas para a saúde pública e falavam da importância da construção de uma proposta de gestão social; descreviam a historicidade das práticas sanitárias no Estado de São Paulo e sua articulação com o trabalho de gerência da enfermagem, alguns estudos discutiam o trabalho de gerencia como uma contribuição para a implantação do SUS nos municípios brasileiros; descreviam o papel da enfermagem na construção dos Sistemas Locais de Saúde e a participação da Enfermagem em todos os níveis de definição das políticas de saúde, buscavam discutir a ética e a cidadania como propostas políticas para direcionarem a gerência de serviços e discutiam o processo de capacitação de gestores de serviços públicos de saúde ${ }^{(20-28)}$. 
Entende-se que, abordar o tema da gerência em saúde a partir de sua dimensão política é uma tarefa complexa, pois exige que este processo de trabalho desenvolva ações nos âmbitos estrutural (relacionadas ao valor e legitimidade social da área da saúde) e político (enquanto equilíbrio de interesses de grupos sociais, visando priorizar a saúde), assim como, a satisfação das demandas operacionais por saúde. Assim, atuar na prática política da gerência deve ser entendido como agir, baseado na decorrência do quanto é possível explicar e compreender a dinâmica das necessidades de saúde e do tipo de explicação formulados acerca da maneira como estão disponíveis e utilizados os recursos, as bases tecnológicas, os interesses e a forma de combiná-los de modo eficaz, eficiente e efetivo $^{(16)}$. Ou seja, falar em gerência de serviços de saúde pressupõe falar da articulação entre necessidades e política publica para satisfazê-las.

A dimensão organizacional do trabalho de gerência, ao ser discutida nos trabalhos científicos trata de discutir como as organizações de saúde, construídas principalmente sob a lógica da hierarquização das funções e dos Processos de Trabalho, conseguem assimilar propostas gerenciais centradas em outras lógicas, como por exemplo, o uso da administração estratégica na perspectiva da gerência de Unidade de Saúde; o planejamento estratégico como possibilidade para transformação da realidade, a construção de programas de saúde que envolva três grandes questões: a qualidade, a missão e o poder institucional; a interpretação do mundo gerencial da enfermagem à luz coerente da liderança transformacional e da inteligência emocional como novos paradigmas para a administração em saúde; a necessidade de se identificar as características pessoais e os estados gerenciais desenvolvidos por enfermeiras de Saúde Pública, a evolução das teorias administrativas e o seu paralelo com o desenvolvimento da assistência à saúde da população, a visão que as instituições de saúde tem do enfermeiro ao atribuir-lhe principalmente um papel de gerência, a tentativa de descrever experiências de gerenciamento que alteram o organograma das organizações de saúde $e^{(29-35)}$.

Alguns estudos articulavam no seu entendimento do trabalho de gerência duas ou mais das dimensões descritas anteriormente. Estes trabalhos examinaram as possíveis modificações ocorridas na assistência de enfermagem desde a implantação do SUS; trazem um relato sucinto sobre a implantação da "Rede Nacional de Informação Bibliográfica e Administração de Serviços de Saúde" - Rede AdSaúde; apresentam elementos para se repensar o planejamento e a administração dos serviços de saúde sob diferentes ângulos; analisam a origem e o contexto em que surge $o$ conceito de "competição estratégica" evidenciando a necessidade de repensar a intervenção estatal no âmbito do SUS; descrevem e analisam fundamentos da Teoria Geral da Administração articulados à prática administrativa da enfermeira na Rede Básica de Saúde e na Rede Hospitalar e traçam um perfil básico das gerentes em um determinado Hospital no município de São Paulo, caracteriza suas atitudes e seus conhecimentos com relação à própria instituição, outros serviços do sistema de saúde e com relação aos princípios do SUS ${ }^{(36-41)}$.

Não foram encontrados trabalhos que buscassem explicar e analisar o trabalho de gerência a partir do olhar tridimensional proposto por Merhy ${ }^{(2)}$, de certa forma isso era esperado, pois essa forma de conceber a gerência é posterior à produção bibliográfica em estudo, contudo é possível identificar estudos que buscavam abordar de maneira ampliada a questão da gerencia dos serviços de saúde, apontando para a insuficiência das abordagens muito técnicas ou muito político-organizacionais.

No campo científico da enfermagem, temas relacionados às questões gerenciais são freqüentemente encontrados uma vez que as funções administrativas estão fortemente relacionadas ao trabalho da enfermeira. Autores como Kurcgant ${ }^{(42)}$, Gomes ${ }^{(43)}$, Novakoski $^{(44)}$, Silva ${ }^{(45)}$, Almeida ${ }^{(46)}$ Mishi$\mathrm{ma}^{(47)} \mathrm{e}$ Fracolli ${ }^{(48)}$ têm produzido importantes estudos nessa área. A leitura desses trabalhos revela que, de modo geral, esses autores entendem que o trabalho de gerência desenvolvido pela enfermeira é fortemente influenciado pelas concepções de organização do trabalho baseadas nos princípios de Taylor, Fayol e nas Teorias Administrativas Clássicas, contudo, essas autoras identificam que propostas gerenciais baseadas nas concepções de organização tecnológica do trabalho; gerência participativa e comunicativa; na teoria das organizações e no pensamento estratégico vêm cada vez mais conquistando espaços nos trabalhos desenvolvidos dentro do campo científico da enfermagem.
Processo de trabalho de gerência: uma revisão de literatura 


\section{CONCLUSÕES}

De uma forma geral o conjunto de trabalhos analisados revelou que, compreendida segundo uma concepção mais abrangente, a prática de gerenciamento de serviços de saúde prescinde de produções científicas que adotem outros marcos teóricos que não aqueles tradicionalmente adotados pela Teoria Geral da Administração, quando administrar poderia estar expresso por adequadas e eficientes combinações entre recursos físicos, materiais, financeiros e humanos.

A teoria que interessa para compreender e formular uma proposta para a gerência de serviços adequada à implantação do SUS dialoga muito pouco com o enfoque hegemônico das propostas administrativas produzidas pela Teoria Geral da Administração. Não que as técnicas de administração estejam superadas, mas porque as características do Movimento Sanitário e sua vinculação com um projeto societário exigem procedimentos que publicizem a gestão dos serviços de saúde de forma a construir um modelo de saúde que defenda a vida dos cidadãos ${ }^{(49-52)}$.

A gestão de um serviço de saúde é uma propriedade de cada um dos agentes do trabalho desse serviço, e de todos ao mesmo tempo, e gerir é operar com o jogo de disputas desses distintos agentes mais do que buscar a funcionalidade não cumprida. Gerir um serviço de saúde é desenvolver intervenções que possibilitem "publicizar" os processos de disputa entre os agentes e a instituição e revelar a "contratualidade" que estes instituem entre $\mathrm{si}^{(2)}$.

Para realizar tal intervenção o gerente necessita utilizar dispositivos de intervenção, capazes de atuar no autogoverno dos profissionais de saúde para poder alterar a lógica do cuidado à saúde e, consequentemente, o modelo de atenção a partir do interior dos serviços.
A incorporação de dispositivos gerenciais que permitam à enfermeira intervir no autogoverno dos trabalhadores pode representar um empowerment na prática administrativa das mesmas. Entende-se que alguns desses dispositivos podem ser :

- procurar valorizar a formação de unidades de saúde baseadas em equipes autônomas, do ponto de vista da gestão do processo de trabalho, dentro de um conjunto de lemas estratégicos definidos na política governamental e social, para garantia do: acesso, acolhimento, vínculo/responsabilização, resolutividade e efetividade, no intuito de alterar a estrutura de necessidades no processo de relação entre usuários e trabalhadores de saúde;

- adotar uma posição na organização de luta pela instituição de unidades de saúde co-gerenciadas politicamente e autogeridas tecno-assistencialmente, buscando a construção de uma rede de serviços estruturada conforme uma lógica horizontalizada de relações de ajuda entre os diferentes estabelecimentos, de acordo com os tipos de incorporações tecnológicas;

- entender que as tecnologias gerencias são importantes, mas, para serem efetivas na transformação dos processos e produtos do trabalho em saúde, precisam estar apoiadas em dimensões político-ideológicas e não apenas em instrumentais técnicos.

- operar com o desenvolvimento de conhecimentos epidemiológicos para a assunção do planejamento, coordenação e avaliação dos programas de saúde de forma ativa.

- desenvolver projetos de pesquisa e produções bibliográficas para o desenvolvimento de temáticas sobre gerenciamento que incorporem as questões acima na pesquisa e na prática da enfermeira.

\section{REFERÊNCIAS}

(1) Campos GWS, Merhy EE, Nunes ED. Plane-jamento sem normas. São Paulo: HUCITEC, 1994.

(2) Merhy EE, Onocko R, organizadores. Agir em saúde: um desafio para o público. São Paulo: HUCITEC; 1997.
(3) Villa TCS. Enfermeira nos serviços de saúde pública do Estado de São Paulo (1967-1983). [Tese] Ribeirão Preto (SP): Escola de Enfermagem de Ribeirão Preto/USP;1992.

(4) Bardin L. Análise de Conteúdo. Lisboa: Edições 70; 1979. 
(5) Braverman H. Trabalho e capital monopolista: a degradação do trabalho no século XX. Rio de Janeiro: Guanabara - Koogan, 1987.

(6) Chiavenato I. Introdução à teoria geral da administração. São Paulo: Mc Graw-Hill do Brasil, 1983.

(7) Castro ML, Ruben G, Serva M. Resíduos e complementaridade: das relações entre teoria da administração e a antropologia. Rev adm publ 1996; 30: 68-80.

(8) Cecílio LCO, organizador. Inventando a mudança na saúde. São Paulo: HUCITEC, 1994.

(9) Mendes VLPS. Gerenciando qualidade em serviços de saúde. Rev Bras Enferm 1993; 6: 211-25.

(10) Guimarães EMP. Sistemas de informação: subsídios para organização e utilização na coordenação da assistência de enfermagem. [Dissertação] Belo Horizonte (MG): Escola de Biblioteconomia da Universidade Federal de Minas Gerais; 1995.

(11) Secretaria Municipal de Saúde de Salvador. Registro de normas, procedimentos e rotinas dos serviços administrativos e técnicos. Salvador; 1996.

(12) Nóbrega MM, Lopes Neto D, Santos SR. Uso da técnica de brainstorming para tomada de decisões na equipe de enfermagem em saúde pública. Brasília. Rev Bras de Enferm 1997; 50: $247-58$

(13) Waldman EA, Rosa TEC. Vigilância em saúde pública. São Paulo: Banco Itaú, 1998.

(14) Collet N, Cesarino CB, Santos, IF. Método funcional na administração em enfermagem: relato de experiência. Rev Bras Enferm 1994; 47: $258-64$.

(15) Carvalho AO, Eduardo MBP. Sistemas de informação em saúde para municípios. São Paulo: Instituto para o Desenvolvimento da Saúde/Faculdade de Saúde Pública/USP; 1998 (Saúde \& Cidadania, 6).

(16) Ferraz CA. A transfiguração da administração em enfermagem da gerência científica para a gerencia sensível. [Tese] Ribeirão Preto (SP): Escola de Enfermagem de Ribeirão Preto/ USP; 1995.

(17) Ribas-Gomes EL. Saber gerir e gerir com saber: a indeterminação do conhecimento administrativo dos enfermeiros. [Livre Docência] Ribeirão Preto (SP): Escola de Enfermagem de Ribeirão Preto/USP; 1999.
(18) Villa TCS, Pinto IC, Mishima SM, Assis MMA. Gerencia do programa de imunização em unidade de saúde: uma proposta de ensino. In: Livro Resumo do $5^{\circ}$ Congresso Paulista de Saúde Publica;1997 jul 3-9; Aguás de Lindóia: ABRASCO, 1997. p.188

(19) Almeida MCP, Luongo J. Buscando a equidade nos serviços de saúde- o papel da gerencia nas unidades básicas de saúde. In: Livro Resumo do $3^{\circ}$ Simpósio de Iniciação cientifica da EERP-USP; 1996 nov. 20; Ribeirão Preto: EERP/USP, 1996.

(20) Merhy EE, Cecílio LCO, Nogueira RC. Por um modelo tecno-assistencial da política de saúde em defesa da vida. In: $9^{\circ}$ Conferência Nacional de Saúde; 1992 out. 10-13; Brasília: GTTCO; 1992. p.30.

(21) Komatsu S. Subdesenvolvimento institucional e políticas sociais: o caso da saúde pública institucional. [Dissertação] São Paulo (SP): Escola de Administração de Empresas de São Paulo; 1993.

(22) Lima SML, Barbosa PR. Gestão em saúde: bases para maior responsabilidade, eficiência e eficácia. Rev Esp Saúde 1996; 5:5-12.

(23) Tanaka OY, Nemes, MIB, Novaes MD, Bastos MG, Cesar CLG, Riedel LF. Formação de gestores locais de saúde: processos para identificar estratégias de atuação. Rev Saúde Pública 1999; 33: 219-229.

(24) Mishima SM, Almeida MCP, Pinto IC, Villa, TCS, Anselmi, ML. Reflexões acerca da gerencia do cuidado de enfermagem na rede básica de saúde. In: Anais no $50^{\circ}$ Congresso Brasileiro de Enfermagem; 1998 out. 10 -13; Salvador: ABEn; 1998. p.179.

(25) Hortale, VA, Conill EM, Pedroza M. Desafios na construção de um modelo para análise comparada da organização de serviços de saúde. Cad saúde publ 1999; 15: 79-88.

(26) Fortes PAC. Ética, cidadania e busca da qualidade na administração dos serviços de saúde. Saúde debate 1996; 49: 48-52.

(27) Dowbor L. Tendências da gestão social. Saúde soc. 1999; 8: 3-16.

(28) Almeida ES, Castro CGJ, Vieira CAL. Distritos Sanitários: concepção e organização. São Paulo: Instituto para Desenvolvimento da Saúde/Faculdade de Saúde Pública/USP (Saúde \& Cidadania, 1).

(29) Sacramento F. Equipe multifuncional: uma ferramenta de produtividade e competitividade. Mundo Saúde 1996, 8: 274-9.
Processo de trabalho de gerência: uma revisão de literatura 
Regina Célia Ermel Lislaine Aparecida Fracolli
(30) Lopes Neto D. Administração pela qualidade: um novo paradigma para a administração dos serviços de enfermagem de saúde pública. [Dissertação] João Pessoa (PB): Centro de Ciências da Saúde da Universidade Federal da Paraíba; 1997.

(31) Régis Filho GI, Lopes MC, Michels G. Democratização da administração em serviços de saúde pública do Taylorismo à gestão participativa. Rev Ciência Saúde 1998, 17: 13-29.

(32) Kisil M, Pupo TRGB. Gestão da mudança organizacional. São Paulo: Instituto para o Desenvolvimento da Saúde/Faculdade de Saúde Pública/USP, 1998. (Saúde \& Cidadania, 4).

(33) Zobolli ELCP. Ética empresarial: de uma conceituação à liderança no ambiente de trabalho. Mundo Saúde 1998, 5: 314-8.

(34) Bittar OJNV. A administração do hospital no próximo milênio. Mundo Saúde 1997; 21:136-41.

(35) Pedersoli CE, Antonialli E, Villa TCS. O enfermeiro na vigilância epidemiológica no município de Ribeirão Preto, 1988-1996. Rev Lat Am Enferm, 1998; 6: 99-105.

(36) Villa TCS, Rocha SMM, Mishina SM, Anselmi ML, Ferrazi C, Almeida MCPA. Caracterização da prática administrativa do enfermeiro nas instituições de saúde. In: Anais do $5^{\circ} \mathrm{Se}-$ minário Nacional de Pesquisa em Enfermagem, 1988 jul 24-9; Belo Horizonte. ABEn. 1990.

(37) Ruiz EAC. Cultura institucional e a viabilização do Sistema Único de Saúde. (SUS): papel dos gerentes de hospital. [Tese] São Paulo(SP): Faculdade de Saúde Pública da USP; 1992.

(38) Ugá MAD. Competição administrativa pelo Estado: notas para discussão. Saúde debate 1995, 46: 14-8.

(39) Andrade MTD, Camargo CA, Sadi BSC. Literatura brasileira na área de administração em saúde em rede Cooperativa. In: Anais do $5^{\circ}$ Congresso da Sociedade Latino-Americano de Estudos sobre América Latina e Caribe; 1996 abr 18-21; São Paulo: 1996. PROLAMP/USP. 1998. P. 252-5.

(40) Mello MR. et. al. O sistema único de saúde e as ações do enfermeiro em uma instituição hospitalar brasileira. Rev. Panam. Salud Publica 1999, 6: 408-14.

(41) Campos GWS. Equipes de Referencia e apoio especializado matricial: um ensaio sobre a reorganização do trabalho em saúde. Ciênc saúde coletiva 1999; 4: 393-403.

(42) Kurcgant P. Administração em enfermagem. São Paulo: EPU;1991.
(43) Ribas-Gomes EL. Administração em enfermagem: constituição histórico-social do conhecimento. [Tese] Ribeirão Preto (SP): Escola de Enfermagem de Ribeirão Preto/ USP, 1991.

(44) Novakoski LRE, Wolf LDG. Gerência participativa: uma proposta para mudança de ambiente no serviço de enfermagem. Rev Bras Enferm, 1992; 45: 187-199.

(45) Silva EM, Gomes EL, Anselmi ML. Enfermagem: realidade e perspectiva na assistência e no gerenciamento. Rev Lat Am Enferm. 1993; 1:59-63.

(46) Almeida MCP. O trabalho de enfermagem e sua articulação com o processo de trabalho em saúde coletiva: rede básica de saúde em Ribeirão Preto. [Livre Docência] Ribeirão Preto (SP): Escola de Enfermagem de Ribeirão Preto/USP; 1991.

(47) Mishima SM Constituição do gerenciamento local na rede básica de saúde Ribeirão Preto. [Tese] Ribeirão Preto (SP): Escola de Enfermagem de Ribeirão Preto/USP;1995.

(48) Fracolli LA. Processo de trabalho de gerência: possibilidades e limites frente à reorganização de rede básica de saúde de Marília. [Tese] São Paulo (SP): Escola de Enfermagem da USP; 1999.

(49) Wirchr P, Mishima SM, Villa TCS, Sá LD. Ensino de gerência de enfermagem sob a ótica do alunoa experiência da territorialização. In: Livro resumo do $48^{\circ}$ Congresso Brasileiro de Enfermagem; 1996 nov 3 -7; São Paulo: ABEn.1996. p.269.

(50) Westrupp MHB, Carraro TE, Souza ML. A pesquisa na enfermagem. Texto Contexto Enferm 1994, 3: 128-36.

(51) Organización Panamericana de la Salud. Reunión del Grupo de Consulta sobre el Papel de Enfermería en el Desarrollo de los Silos: informe final. Guatemala;1992.

(52) Villa TCS. Ensino de enfermagem em Saúde Publica: da normatização à gerência participativa. In: Anais $5^{\circ}$ Congresso Paulista de Saúde Publica;1997 jul 3-9; Águas de Lindóia. ABRASCO, 1997. p.188 